\title{
Pensamiento matemático y creatividad a través de la invención y resolución de problemas matemáticos
}

\author{
Mathematical thinking and creativity through \\ mathematical problem posing and solving
}

\section{María F. Ayllón ${ }^{12 a}$, Isabel A. Gómez ${ }^{12 b}$ \& Julio Ballesta-Claver ${ }^{12 b *}$ \\ ${ }^{1}$ Centro de Magisterio La Inmaculada, Granada, España. ${ }^{2}$ Universidad de Granada, Granada, España.}

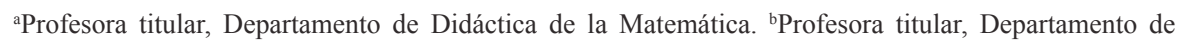
Psicología. 'Profesor, Departamento de Ciencias Experimentales.

Recibido: $30-07-15$

Aprobado: 23-11-15

\section{*Correspondencia}

Email: juliosci@eulainmaculada.com

\section{Citar como:}

Ayllón, M., Gómez, I., \& Ballesta-Claver, J. (2016). Pensamiento matemático y creatividad a través de la invención y resolución de problemas matemáticos. Propósitos y Representaciones, 4(1), 169-218. doi: http://dx.doi.org/10.20511/pyr2016. v4n1.89

(C) Universidad San Ignacio de Loyola, Vicerrectorado de Investigación y Desarrollo, 2016. (c) BY-NC-ND Este artículo se distribuye bajo licencia CC BY-NC-ND 4.0 Internacional (http://creativecommons.org/licenses/by-nc-nd/4.0/). 


\section{Resumen}

En este trabajo se muestra la relación entre el desarrollo del pensamiento matemático y la creatividad con la invención y resolución de problemas matemáticos. No son frecuentes las ocasiones en las que aparecen juntas Creatividad y Matemáticas. Ambas constituyen procesos complejos que comparten elementos como la fluidez (número de ideas), la flexibilidad (variedad de ideas), la novedad (idea única) y la elaboración (desarrollar una idea). Estos factores contribuyen, entre otras cosas, a que los escolares sean competentes en matemáticas. La invención y la resolución de problemas resultan ser un instrumento evaluador muy potente con el que se pone de manifiesto el nivel de razonamiento matemático y creativo de una persona. La creatividad forma parte de la educación matemática y constituye un ingrediente necesario para realizar las tareas matemáticas. Esta aportación presenta algunas investigaciones relevantes, que se han llevado a cabo sobre invención y resolución de problemas, vinculadas al desarrollo del conocimiento matemático y a la creatividad. Para ello se parte de las distintas creencias que se recogen en la literatura referente a las nociones de creatividad, invención de problemas y resolución de problemas.

Palabras clave: Resolución de problemas, invención de problemas, Educación Matemática y creatividad.

\section{Summary}

This work shows the relationship between the development of mathematical thinking and creativity with mathematical problem posing and solving. Creativity and mathematics are disciplines that do not usually appear together. Both concepts constitute complex processes sharing elements, such as fluency (number of ideas), flexibility (range of ideas), novelty (unique idea) and elaboration (idea development). These factors contribute, among others, to the fact that schoolchildren are competent in mathematics. The 
problem solving and posing are a very powerful evaluation tool that shows the mathematical reasoning and creative level of a person. Creativity is part of the mathematics education and is a necessary ingredient to perform mathematical assignments. This contribution presents some important research works about problem posing and solving related to the development of mathematical knowledge and creativity. To that end, it is based on various beliefs reflected in the literature with respect to notions of creativity, problem solving and posing.

Keywords: Problem solving, problem posing, mathematics education and creativity. 


\section{Introducción}

El principal objetivo de la enseñanza de las matemáticas es desarrollar el pensamiento. Una vía que ayuda a alcanzar dicho objetivo es realizar tareas de invención y resolución de problemas. Cuando un individuo se enfrenta a la tarea de inventar un problema, se ve obligado a pensar, a analizar críticamente el enunciado, a examinar los datos que este presenta y a manipular distintas estrategias de resolución que permitan obtener la solución de dicho problema. Todo esto también se ejercita a través de la resolución de problemas. La resolución y la invención de problemas ayudan a afianzar lo que se está aprendiendo. En este sentido, Noda (2000) manifiesta que una y otra actividad son fundamentales para la construcción del conocimiento matemático constituyendo, tanto para la teoría como para la práctica educativa, una acción cognitiva básica esencial. Por ello, se considera que el concepto de número y la resolución e invención de problemas constituyen el eje vertebrador de los conocimientos matemáticos escolares (Ayllón, 2012).

La resolución de problemas es un componente básico para el aprendizaje, así como para la adquisición del conocimiento. García (1998) considera que inventar problemas es prioritario para consolidar y avanzar en el conocimiento. También advierte que los investigadores pueden alcanzar logros a través de sus teorías científicas al formular, descubrir o enfrentarse a campos problemáticos novedosos.

Cuando una persona se enfrenta a resolver un problema, a priori, le supone una tarea no demasiado fácil, constituyendo además un desafío con el que desarrollará su creatividad y sus habilidades matemáticas.

Esta circunstancia también se encuentra en el planteamiento de problemas. La invención de problemas permite adquirir aprendizajes significativos e indaga en las capacidades matemáticas que la persona tiene, al establecer relaciones entre los distintos conceptos matemáticos, así como 
en las estructuras numéricas. La invención de problemas requiere poseer un nivel de abstracción elevado y obliga a reflexionar, permitiendo alcanzar así una fase de razonamiento que facilita la construcción del conocimiento matemático. La persona que inventa un problema matemático parte de sus ideas propias, por lo que sigue un proceso creativo. Por tanto, se considera que estamos ante una situación de invención de problemas cuando esta es una producción propia y no una reformulación de un problema ya planteado.

Desde el momento de su nacimiento, las personas buscan explicaciones a todo lo que les rodea poniendo a funcionar su creatividad. Dicha creatividad hay que desarrollarla, estimularla y fomentarla, por lo que es necesario educarla e incluirla en los centros escolares. Barbarán y Huguet (2013) consideran que el sistema educativo actual no fomenta la creatividad. Afirman que incluso, en ocasiones, la obstaculiza.

Distintos investigadores relacionan la invención de problemas con el desarrollo del conocimiento matemático y de la creatividad. Autores como Krutetskii (1969) y Ellertoh (1986) apuntan la existencia de una relación implícita entre la habilidad que se requiere para inventar problemas y el nivel de creatividad, así como de su competencia matemática. También se encuentra una evidente relación entre la creatividad y la resolución de problemas (Callejo, 2003) debido a que, cuando una persona se halla en situación de resolver un problema, se aviva su creatividad al realizar dicha tarea. Ayllón y Gómez (2014) manifiestan que las tareas de invención de problemas desarrollan la creatividad en los escolares y mejoran la adquisición de conceptos matemáticos.

\section{Invención de problemas.}

A lo largo de la literatura científica, distintos estudios abordan el campo de la invención de problemas manifestando que se trata de una tarea fundamental para adquirir el conocimiento matemático (Stoyanova \& Ellerton, 1996; Nicolaou 
\& Pilippou 2007; DeHaan, 2009; Kesan, Kaya \& Guvercin, 2010; Ayllón, 2012; Fernández \& Barbarán, 2012; Pintér, 2012; Espinoza \& Segovia, 2013; Fernández, 2013; Ayllón \& Goméz, 2014). Es cierto que las investigaciones en invención de problemas no son tan frecuentes ni presentan la larga trayectoria investigadora del campo de la resolución de problemas. No obstante, desde las últimas décadas son cada vez más los autores que abordan este campo.

Los investigadores se refieren al término invención de problemas de formas variadas. Así se encuentran términos como: problemas (Kilpatrick, 1987), planteamiento de problemas (Brown \& Walter, 1993), formulación y generación de problemas (Silver, 1994). Estos términos también dan paso a la formulación de nuevos problemas o a la reformulación de situaciones dadas (Silver \& Cai, 1996). Es decir, se puede inventar un problema durante la solución de este (Silver, Mamona-Down, Leung \& Kenny, 1996) mediante cambios que se realicen al mismo, reformulándolo y/o centrándose en un caso concreto, con la finalidad de entender mejor el problema y, así, poder resolverlo. También se puede inventar un problema una vez que ya se ha resuelto uno, modificando los objetivos, condiciones y/o preguntas del mismo (Silver, 1994). En este sentido, Brown y Walter (1993) explican cómo plantear nuevos problemas a partir de uno dado siguiendo la estrategia "¿What if not?", que consiste en modificar las condiciones de un problema determinado.

Por su parte, Shukkwan (1993) propone la formulación de problemas a partir de una solución dada, y Mamona-Downs (1993), como la actividad que resulta cuando un problema invita a la generación de otros problemas.

Espinoza, Lupiañez y Segovia (2013) recogen distintas maneras de formular problemas matemáticos: situación libre, situaciones semiestructuradas y situaciones estructuradas (Stoyanova, 1998). La situación libre no requiere condición alguna para inventar un problema; la 
semiestructurada requiere una situación concreta de partida, y la estructurada es aquella en la que se modifica alguna condición de un problema dado.

La invención de problemas exige realizar una aportación personal, propia y creativa, además de utilizar el conocimiento matemático ya adquirido y de relacionar distintos conceptos. Cuando se inventa un problema, se parte de situaciones concretas e interpretaciones personales (Koichu \& Kontorovich, 2012).

En el año 2005, Christou, Mousoulides, Pittalis y Pitta-Pantazi realizaron un estudio que puso de manifiesto que la invención de problemas favorece el aprendizaje matemático. En él indican que una persona, al inventar un problema matemático, está obligada a establecer relaciones entre distintos conceptos matemáticos e involucra distintos procesos cognitivos, como editar, seleccionar, comprender, organizar y traducir la información de una forma de representación a otra.

Barbarán y Huguet (2013) realizaron un estudio con alumnos de $1^{\circ}$ de Educación Secundaria Obligatoria donde evidencian que las tareas de invención y reconstrucción de problemas matemáticos fomentan el desarrollo de la creatividad. Afirman que estas impulsan, de forma activa, la creatividad de los escolares al obligarlos a proyectar sus ideas generando problemas que sean originales. También apuntan que la invención de problemas favorece que el profesorado utilice una metodología donde el protagonista principal de su propio aprendizaje es el alumno, promoviendo así el desarrollo de su creatividad.

Ayllón y Gómez (2014) recogen los beneficios que reporta la tarea de inventar problemas, haciendo una recopilación de las aportaciones de distintos investigadores y categorizando tales aspectos en seis factores positivos: 
1. El aumento del conocimiento matemático y lingüístico. Inventar un problema exige una redacción clara del enunciado, de forma organizada y exacta. Requiere que se analicen los datos y se razone críticamente, se discutan y cuestionen ideas y soluciones. Por tanto, el inventor ha de escribir con claridad, exactitud y organización (Davidson \& Pearce, 1988; Burçin, 2005; Whitin, 2006).

2. El incremento de la motivación. La invención de problemas estimula la curiosidad y la motivación en los escolares (Silver, 1994; Akay \& Boz, 2010).

3. La disminución de la ansiedad que en determinadas ocasiones padecen algunos escolares origina su relación con las matemáticas. Autores como Burçin (2005) y Song, Yim, Shin y Lee (2007) afirman que, cuando el estudiante realiza tareas de invención de problemas, disminuye su miedo a las matemáticas.

4. El vencimiento de errores matemáticos frecuentes por parte de los escolares. Brown y Walter (1993) manifiestan que la invención de problemas merma los fallos y errores resolutivos que cometen los estudiantes debido a que eligen de forma apropiada la información, así como los datos para la resolución del problema.

5. El aumento de la creatividad. Autores como Krutetskii (1969), Ellertoh (1986) y Silver (1994) evidencian el vínculo entre la habilidad para inventar problemas y el nivel de creatividad y de competencia matemática.

6. Como herramienta evaluadora. La invención de problemas es un instrumento evaluador para el profesorado. Mediante esta tarea, el docente puede conocer mejor los conceptos adquiridos y a sus alumnos (Lin, 2004; Ayllón, 2005; Sheikhzade, 2008), pudiendo valorar el tipo de razonamiento matemático que estos poseen.

\section{Resolución de problemas.}

Se ha comentado que la literatura científica se refiere a la invención de problemas con distintas acepciones; de igual forma encontramos que 
los investigadores identifican la resolución de problemas con diferentes consideraciones. Koestler (1964) vincula la resolución de problemas a la creatividad. Afirma que, cuando una persona resuelve un problema, este supone un aporte a la chispa de la creatividad. Agre (1982) considera que la resolución de problemas corresponde a un proceso en el que se utilizan conocimientos que ya han sido adquiridos a través de una situación novedosa y que no es familiar. Por otra parte, Puig (1993) alega que la resolución de problemas constituye una actividad mental que se produce desde el momento en que al resolutor se le propone un problema, siendo consciente este de que lo que se le ha presentado es un problema, sintiendo la necesidad de resolverlo y terminando su actividad una vez que lo ha solucionado. Contreras (1998) manifiesta que la resolución de problemas corresponde a la situación en la que una persona que se enfrenta a un problema es capaz de comprenderlo, ya que posee previamente un aprendizaje, pero no sabe en ese instante cómo resolverlo, aunque trata de buscar su solución enfrentándose a dicha situación. Contreras también afirma que la resolución de problemas corresponde a una tarea meramente perceptiva y conceptual.

García (1998) recoge cinco líneas de investigación en el campo de la resolución de problemas: la resolución de problemas como estrategia para generar cambios conceptuales, metodológicos y actitudinales; la organización cognoscitiva del conocimiento y la capacidad para resolver problemas; la comparación entre individuos expertos y novatos; el diseño de heurísticos para la resolución de problemas, y la creatividad como resolución de problemas. Esta última línea entiende que la resolución de problemas conlleva procesos en los que es necesario modificar una situación generando ideas creativas, por lo que considera que la creatividad es una forma de resolución de problemas y viceversa; es decir, la resolución de problemas es una manera eficaz de desarrollar la creatividad.

Schroeder y Lester (1989) diferencian el trabajo de la resolución de problemas en el aula en tres categorías para enseñar: sobre resolución 
de problemas, para resolver problemas o vía resolución de problemas. Consideran que enseñar sobre resolución de problemas corresponde a trabajar estrategias de resolución; es decir, el fin es que el estudiante sepa resolver problemas. Al referirse a enseñar para resolver problemas, hablan de cómo utilizar los conocimientos matemáticos ya adquiridos en la resolución de problemas. La última categoría, la enseñanza vía resolución de problemas, radica en partir de problemas con el objeto de aprender nuevos conocimientos matemáticos. Estos tres objetivos son distintos: el primero tiene por finalidad que los escolares sepan resolver problemas; el segundo, que afiancen sus conocimientos, y el tercero, utilizar una metodología cuyo instrumento es la resolución de problemas.

Uno de los marcos idóneos para la construcción de un aprendizaje significativo es la resolución de problemas, ya que contribuye a aumentar el gusto por la matemática y fomenta el desarrollo de una actitud crítica y abierta (Carrillo, 1996). Como ya se ha dicho, una persona que se enfrenta a un problema está aprendiendo y dando uso a los conceptos matemáticos ya adquiridos.

Autores como Polya (1965) afirman que un barómetro del conocimiento matemático es la resolución de problemas. Junto con este mismo autor, Gagné (1965) y Brown (1978) sostienen que la resolución de problemas no solo se vale de conocimientos adquiridos, sino que genera conocimientos nuevos.

Sin embargo, para saber resolver problemas, previamente hay que aprender. Brown (1978) asegura que la resolución de problemas es un aprendizaje, del mismo modo que lo son la memorización simple, el aprendizaje algorítmico y el aprendizaje conceptual, y afirma que la resolución de problemas constituye una herramienta para la construcción y adquisición de los aprendizajes señalados. 


\section{Creatividad.}

La evolución de un país depende del desarrollo científico del mismo, y para ello se necesitan personas creativas. Ramos (2006, p. 9) dice que "educar para ser creativos es un requisito esencial en los inicios del siglo XXI".

La Real Academia Española (22 ${ }^{\mathrm{a}}$ ed.) define creatividad como "la facultad de crear, capacidad de creación". A lo largo de la literatura no se encuentra un acuerdo referente a la noción de creatividad. Stein (1956) se refiere a la creatividad como aquel proceso que culmina en una obra nueva resultando útil; De Bono (1974) manifiesta que la creatividad es una actitud mental y un instrumento de pensamiento, por lo que se trata de una forma de utilizar la mente y la información; De la Torre (1984) opina que se trata de una aptitud, mientras que Goleman, Kaufman y Ray (1992) afirman que la creatividad es una actitud. Sin embargo, Sorin (1992) dice que toda persona puede ser considerada creativa, siempre que sea singular en su campo y produzca innovaciones.

Otras definiciones vinculan la creatividad a un estilo de pensamiento. También están las que se basan en procesos creativos, mientras que otras lo hacen en productos creativos. Nadjafikhah y Yaftian (2013) afirman que el pensamiento creativo es un proceso mental dinámico que engloba los pensamientos divergente y convergente. El pensamiento divergente contiene cuatro elementos necesarios para considerar que una producción es creativa: fluidez (número de ideas), flexibilidad (se acerca a la variedad de ideas), novedad (idea única) y elaboración (desarrollar una idea).

Por tanto, la creatividad puede entenderse y definirse de distintas formas, aunque se puede resumir como una actividad personal o grupal destinada a producir algo nuevo. No obstante, la definición que tiene mayor reconocimiento es la manifestada por el National Advisory Committee on Creative and Cultural Education (NACCCE, 1999, p. 30), que se refiere a 
la creatividad como "cualquier actividad imaginativa cuyo fin consiste en producir resultados que son, a la vez, originales y de alto valor". Bolden, Harries y Newton (2010) sostienen que esta referencia a la "originalidad" y "valor" tiene implícito un aspecto social, ya que es la sociedad, en última instancia, la que valida el producto.

García (1998) explica que la creatividad se caracteriza por los siguientes elementos o capacidades:

a. Sensibilidad a los problemas: esta capacidad permite a la persona problematizar situaciones y buscar soluciones a las mismas.

b. Flexibilidad: esta capacidad brinda la posibilidad de cambiar los enfoques de un problema haciendo que la persona busque estrategias diferentes para resolverlo.

c. Fluidez de pensamiento: permite generar ideas en un tiempo determinado.

d. Originalidad: a partir de conocimientos adquiridos se producen conocimientos nuevos.

e. Capacidad para percibir conexiones no obvias entre los hechos: se descubren relaciones no establecidas anteriormente entre experiencias distintas.

f. Capacidad de representación: se establecen nuevos modelos y se descubren relaciones diferentes entre sus elementos.

\section{Creatividad y educación matemática.}

Tradicionalmente, la creatividad solo se atribuía al mundo del arte y de la literatura. Hoy también se vincula al mundo científico. Una idea científica, para ser creativa, ha de ser novedosa y útil. El pensamiento matemático propicia el desarrollo de la creatividad debido a que requiere hacer conjeturas y discernir opciones que permitan resolver una situación planteada. 
En educación matemática, la creatividad se basa en conocimientos. Consiste en construir algo nuevo liberándose previamente de los modos de pensar establecidos, viendo distintas posibilidades y aplicando una gama variada de conocimientos matemáticos (Bolden, Harries \& Newton, 2010). La justificación para llevar a cabo esto es la flexibilidad cognitiva, una de las tres funciones mentales principales que están implicadas en la solución creativa de problemas (Ausubel, 1963, 2000). Por su parte, DeHaan (2009, 2011) afirma que es posible aplicar ideas a contextos nuevos, a lo que se refiere como la "transferencia" de la capacidad de conocimiento, haciendo que se desarrollen activamente las representaciones de los escolares y convirtiendo la información recibida en algo más útil, práctico y constructivo.

Se recogen dos vías de investigación referidas a la creatividad y la educación matemática, las que utilizan la palabra creatividad como sujeto: la creatividad en educación matemática, o como adjetivo: la educación matemática recreativa (Sequera, 2007). Las investigaciones de creatividad en educación matemática consideran la creatividad como un elemento metodológico que ayuda a adquirir el aprendizaje matemático y cercioran que al trabajar la resolución de problemas no solo se desarrollan habilidades de razonamiento, sino también habilidades creativas. En este argumento prevalece la creatividad, a partir de la cual se aprenden las matemáticas. Los estudios que hacen referencia a la educación matemática creativa sostienen que, a partir de la enseñanza de las matemáticas, brota la creatividad. Por tanto, cuando se está en un proceso de resolución de problemas, surge un proceso creativo.

\section{Creatividad e invención de problemas.}

En las investigaciones se encuentran distintos métodos para averiguar cuándo una persona es creativa. Uno de ellos es el utilizado por Getzels y Jackson (1962), donde realizan tareas de invención de problemas con el objetivo de medir la creatividad. Esta misma técnica la emplea Balka en 
1974, estudiando las producciones de estudiantes con base en la originalidad de los enunciados inventados.

Silver (1997) argumenta que cuando se instruye en matemáticas, a partir de tareas de invención de problemas, el profesor ayuda a que los estudiantes desarrollen su creatividad aumentando la capacidad de estos en cuanto a fluidez, flexibilidad y novedad. En esta línea se recogen las aportaciones de otros autores, como Van den Brink (1987), Streefland (1987), Healy (1993) y Skinner (1991), quienes afirman que el planteamiento de problemas fomenta el desarrollo de la fluidez, siendo esta la característica principal de la creatividad.

Singer, Perczer y Voica (2011) analizaron la creatividad de estudiantes, de 11 a 13 años, que inventan problemas. Su investigación evidencia que aquellos estudiantes que inventan problemas coherentes $\mathrm{y}$ originales mediante cambios que realizan a sus enunciados presentan habilidades creativas.

Por su parte, Van Harpen y Sriraman (2013) llevaron a cabo un estudio en China y Estados Unidos con estudiantes de secundaria, a fin de analizar la creatividad de estos. Para ello realizaron actividades basadas en la invención de problemas cuyo contenido matemático debían ser conceptos geométricos. Obtuvieron resultados que constataban que, cuando un alumno plantea un problema matemático, desarrolla habilidades de fluidez. También se evidenció que los escolares enunciaron problemas de alta calidad en cuanto a su elaboración y la originalidad de los planteamientos. Estos autores proponen incluir la invención de problemas en el currículo escolar, dentro de la asignatura de matemáticas, por los beneficios que esta tarea conlleva para los estudiantes.

Dickman (2014) realizó un estudio con el que pretende indagar sobre la conexión existente entre el planteamiento de problemas y la creatividad. 
Para ello pide a un grupo de profesores de primaria, a psicólogos que trabajan en educación matemática y a matemáticos, que evalúen problemas inventados en cuanto a lo creativos que son. Su estudio le lleva a evidenciar que no hay un consenso entre ellos debido a que no comparten la misma definición de creatividad, aunque sí admiten la vinculación entre creatividad e invención de problemas.

\section{Creatividad y resolución de problemas como procesos.}

Muñoz, en 1994, hace una alusión a las investigaciones que se han realizado hasta ese momento acerca de la creatividad, en aspectos como la persona, el proceso, el producto y el ambiente.

La creatividad como proceso hace referencia a las fases necesarias por las que se ha de pasar para alcanzar un producto creativo. En 1908, Poincaré define estas fases y las denomina: preparación, incubación, iluminación. La primera fase corresponde al tiempo inicial de trabajo consciente, siendo este intenso; la segunda fase se refiere a un tiempo de descanso, y la tercera se sitúa en el momento en que aparecen del inconsciente las ideas. En 1992, Goleman, Kaufman y Ray recogen estas fases añadiendo una más a la que denominan "de traducción", explicando que se trata del momento en el que la idea deja de ser pensamiento y se transforma en una realidad. Más tarde, en 1998, García renombra y completa estas fases en: a) encuentro con el problema, fase en la cual el sujeto utiliza su pensamiento crítico, siente la necesidad de crear, solucionar el problema o, incluso, de exteriorizar ideas que le preocupan; b) generación de ideas, en la que, a partir de la inspiración, la persona busca posibles soluciones al problema y, de esta forma, genera una nueva idea; c) elaboración de la idea, cuando se materializa la creación o proyecto, y d) transferencia creativa, que es la última fase del proceso creador y donde se relaciona la nueva idea con otras ya conocidas. 
Se aprecia que estas fases del proceso creativo corresponden a las que concretan Hadamard (1945) y Polya (1965) referentes al proceso de resolución de problemas. Hadamard las define en cuatro: a) trabajo consciente de familiarización con el problema, b) trabajo semiconsciente o inconsciente de incubación de las ideas, c) inspiración o iluminación sobre la forma de resolver el problema, y d) verificación de que la inspiración conduce realmente a la solución. Por otra parte, Polya las define en: a) comprensión del problema, b) concepción de un plan, momento en el que se elabora una estrategia de resolución, c) ejecución del plan, y d) comprobación.

Se observa una coincidencia en las fases necesarias para desarrollar un producto creativo con las requeridas para resolver un problema. Existe una correspondencia entre preparación o encuentro con el problema (creatividad) y comprensión del problema o familiarización con el mismo, debido a que las dos fases hacen referencia al instante preliminar a lo que se va a afrontar. La segunda y tercera fase, incubación e iluminación o generación y elaboración de la idea, se encuentran tanto en el proceso creativo como en el de resolución de problemas, incluso con denominaciones idénticas. La última fase, traducción o transferencia creativa (creatividad) y verificación (problemas), corresponde al momento en el que se ha alcanzado el objetivo propuesto.

\section{Conclusiones}

Hoy en día, los educadores tienen como misión prioritaria identificar y desarrollar la creatividad, y precisan de ambientes adecuados para el aprendizaje creativo. Dicho aprendizaje requiere que sea flexible, asocie materiales e ideas, presente metodologías indirectas, motivantes e imaginativas y favorezca la relación entre alumno-profesor (De la Torre, 1995). A su vez, una de las mayores preocupaciones del profesorado es lograr que la enseñanza-aprendizaje de las matemáticas desarrolle el pensamiento lógico en los escolares mediante metodologías constructivistas y lúdicas, 
para hacer de las matemáticas una herramienta aplicable a la vida diaria. Este aprendizaje, gracias al que se adquiere el conocimiento matemático, está unido a la creatividad del alumno y a su desarrollo.

Tras lo expuesto en los párrafos anteriores, queda evidenciado que existe una vinculación entre la creatividad y la educación matemática. Dicha vinculación concurre en doble sentido. La matemática es un instrumento que permite solventar situaciones problemáticas que surgen en nuestro día a día. Esta herramienta se fundamenta en la creación de nuevos conceptos, de estrategias resolutorias y/o de distintas formas de pensamiento. Por su parte, la creatividad es un instrumento de toda actividad matemática. Vianney y Navarro (2011) afirman que la creatividad precisa que se construyan relaciones entre diversos conceptos con el fin de crear algo novedoso. Para desarrollar adecuadamente el pensamiento matemático, la invención y la resolución de problemas se convierten en tareas fundamentales, conformando así el eje vertebrador del mismo, donde hay que relacionar ideas, asociar conceptos, usar la memoria y utilizar el pensamiento crítico. Por tanto, se parte de la innovación y de la creatividad en las tareas de invención y resolución de problemas.

La creatividad forma parte de la actividad matemática (Sequera, 2007), de forma que está implícita en las actividades de invención y resolución de problemas, las cuales identifican las capacidades matemáticas de los alumnos, convirtiéndolas en un potente instrumento evaluador para los docentes.

Whitin (2006) y Sequera (2007) invitan a que se incluya en las aulas las tareas referidas a invención de problemas, con el objeto de proporcionar a los escolares una educación matemática creativa, es decir, un aprendizaje matemático vivo y rico, donde se cuestione sobre los problemas inventados y sus soluciones, se promuevan estrategias de resolución de problemas que inciten a la imaginación y se desarrolle un pensamiento flexible. Ayllón 
(2005) advierte que en el momento en que un individuo inventa un problema lo siente suyo, creando contextos reales y cercanos a él, aumentando sus ganas de aprender, atreviéndose a cometer errores y aprendiendo de ellos, y mermando el miedo que para algunos estudiantes, en determinados momentos, genera el aprendizaje de las matemáticas. El estudiante ha de hacer uso de su creatividad para plantear un problema matemático.

Actualmente, la enseñanza de las matemáticas, en muchas ocasiones, frena el desarrollo de la creatividad al fijar unas pautas demasiado rígidas de actuación frente a la resolución de problemas. Es necesario fomentar la creatividad de los escolares para mejorar la enseñanza matemática. Los estudiantes han de aprender a pensar, hacer y deshacer libremente para dar rienda suelta a su imaginación y generar cosas nuevas, de la misma forma que cuando construyen un significado para los símbolos, signos y operaciones. Pichel (2001) propone tareas donde los niños reflexionen sobre el número, jueguen a la criptografía, inventen operaciones, estrategias y problemas, analicen juegos, etc. Con ellas se evita repetir siempre los mismos procesos consolidados que impiden desarrollar la creatividad.

Resolver problemas matemáticos e identificar el significado de estos favorece la búsqueda y el desarrollo del razonamiento que dará paso a la creatividad (Haylock, 1987). Todo ello conlleva mejoras en el comportamiento, en las habilidades sociales, la motivación, la autoestima y el rendimiento en todos los aspectos. Por ello, es importante desarrollar estrategias e instrumentos matemáticos que favorezcan el aprendizaje creativo en nuestras aulas. 


\section{Referencias}

Agre, G. P. (1982). The concept of problem. Educational Studies, 13, 121142.

Akay, H., \& Boz, N. (2010). The effect of problem posing oriented analysesII course on the attitudes toward mathematics and mathematics selfefficacy of elementary prospective mathematics teachers. Australian Journal of Teacher Education, 35(1), 59-65.

Ayllón, M. F. (2005). Invención de problemas con números naturales, enteros negativos y racionales. Tarea para profesores de educación primaria en formación. Trabajo de investigación tutelada, Universidad de Granada, España.

Ayllón, M. F. (2012). Invención-Resolución de problemas por alumnos de educación primaria en formación (Tesis doctoral). Universidad de Granada, España.

Ayllón, M. F., \& Gómez, I. A. (2014). La invención de problemas como tarea escolar. Escuela Abierta: Revista de Investigación Educativa 17, 29-40.

Ausubel, D. P. (1963). The psychology of meaningful verbal learning; an introduction to school learning. New York: Grune y Stratton.

Ausubel, D. P. (2000). The acquisition and retention of knowledge: A cognitive view. Boston: Kluwer.

Balka, D. S. (1974). Creative ability in mathematics. Arithmetic Teacher 21, 633-636.

Barbarán, J. J., \& Huguet, A. (2013). El desarrollo de la creatividad a través de la invención de problemas matemáticos. Un estudio con alumnos de secundaria. Revista Internacional de Educación y Aprendizaje, 1(2), 1-9.

Bolden, D. S., Harries, A. V., \& Newton, D. P. (2010). Pre-service primary teachers' conceptions of creativity in mathematics. Educational Studies in Mathematics, 73(2), 143-157.

Brown, M. (1978). Cognitive development and the learning of mathematics. En A. Floyd (Ed.), Cognitive development in the school years (pp. 351373). Londres: Croom Helm. 
Brown, S. I., \& Walter, M. I. (1993). Problem posing. Hillsdale, NJ: Lawrence Erlbaum Associates.

Burçin, B. (2005). The effect of instruction with problem posing on tenth grade students 'probability achievement and attitudes toward probability (Tesis doctoral inédita). Turquía, Universidad de Ankara.

Callejo, M. L. (2003). Creatividad matemática y resolución de problemas. Sigma 22, 25-34.

Carrillo, J. (1996). Modos de resolver problemas y concepciones sobre la matemática y su enseñanza de profesores de matemáticas de alumnos de más de 14 años. Algunas aportaciones a la metodología de la investigación y estudio de posibles relaciones (Tesis doctoral inédita). Universidad de Sevilla, España.

Christou, C., Mousoulides, N., Pittalis, M., \& Pitta-Pantazi, D. (2005). Problem solving and problem posing in a dynamic geometry environment. The Montana Mathematics Enthusiast, 2(2), 125-143.

Contreras, L. C. (1998). Resolución de problemas: un análisis exploratorio de las concepciones de los profesores acerca de su papel en el aula (Tesis doctoral inédita). Universidad de Huelva, España.

Davidson, D., \& Pearce, D. (1988). Using writing activities to reinforce mathematics instruction. Arithmetic Teacher, 35(18), 42-45.

De Bono, E. (1974). El pensamiento lateral. Barcelona: Paidós.

De la Torre, S. (1984). Creatividad plural. Sendas para indagar sus múltiples perspectivas. Barcelona: PPU.

De la Torre, S. (1995). Creatividad aplicada. Madrid: Escuela Española.

DeHaan, R. L. (2009). Teaching creativity and inventive problem solving in science. CBE-Life Sciences Education, 8(3), 172-181.

DeHaan, R. L. (2011). Teaching creative science thinking. Science 334, 1499-1500.

Dickman, B. M. (2014). Conceptions of Creativity in Elementary School Mathematical Problem Posing (Tesis doctoral inédita). Nueva York, Universidad de Columbia. 
Ellertoh, N. F. (1986). Children's made up mathematics problems. A new perspective on talented mathematicians. Educational Studies in Mathematics, 17(3), 261-271.

Espinoza, J., \& Segovia, I. (2013). La invención de problemas como actividad matemática. IV Encuentro de Enseñanza Matemática, UNED 2013 (3031 agosto 2013). San José, Costa Rica.

Espinoza, J., Lupiañez, J. L., \& Segovia, I. (2013). Invención de problemas aritméticos por estudiantes con talento en matemática: un estudio exploratorio. I Congreso de Educación Matemática de América Central y el Caribe (pp. 899-911). Santo Domingo, República Dominicana: Pontificia Universidad Católica Madre.

Fernández, E. (2013). Invención de problemas por estudiantes de secundaria: evaluación de su conocimiento sobre simbolismo algebraico. Trabajo Fin de Máster. Universidad de Granada.

Fernández, J. A., \& Barbarán, J. J. (2012). Incidencia de la invención y reconstrucción de problemas en la competencia matemática. Revista Iberoamericana de Educación Matemática, 32, 29-43.

Gagné, R. (1965). The conditions of learning. New York, NY: Holt, Rinehart y Winston.

García, J. J. (1998). La creatividad y la resolución de problemas como bases de un modelo didáctico alternativo. Revista de Educación y Pedagogía, 10(21), 145-173.

Getzels, J. W., \& Jackson, P. W. (1962). Creativity and intelligence. New York: John Wiley y Sons, Inc.

Goleman, D., Kaufman, P., \& Ray, M. (1992). El espiritu creativo. Barcelona: Vergara.

Hadamard, J. (1945). An essay on the psychology of invention in the mathematical field. New York: Princeton University Press.

Haylock, D. W. (1987). A framework for assessing mathematical creativity in school children. Education Studies in Mathematics, 18(1), 59-74.

Healy, C. C. (1993). Creating miracles: A story of student discovery. Berkeley, CA: Key Curriculum Press. 
Kesan, C., Kaya, D., \& Guvercin, S. (2010). The effect of problem posing approach to the gifted student's mathematical abilities. International Online Journal of Educational Sciences, 2, 677-687.

Kilpatrick, J. (1987). Problem formulating: where do good problems come from? En A. H. Schoenfeld (Ed.), Cognitive science and mathematics education (pp. 123-127). Hillsdale, NJ: Laurence Erlbaum Associate.

Koestler, A. (1964). The act of creation. New York: Macmillan.

Koichu, B., \& Kontorovich I. (2012). Dissecting success stories on mathematical problem posing: a case of the Billiard Task. Educational Studies in Mathematics, 83(1), 71-86.

Krutetskii, V. A. (1969). An investigation of mathematical abilities in schoolchildren. En J. Kilpatrick e I. Wirszup (Eds.), Soviet studies in the psychology of learning and teaching mathematics (Vol. 2, pp. 5-57). Chicago, IL: University of Chicago Press.

Lin, P. J. (2004). Supporting teachers on designing problem-posing tasks as a tool of assessment to understand students' mathematical learning. En M. Hoines y A. Fuglestad (Eds.), Proceedings of the $28^{\text {th }}$ annual meeting of the International Group for the Psychology of Mathematics Education (Vol. 3, pp. 257-264). Bergen, Noruega: Bergen University College.

Mamona-Downs, J. (1993). On anaysing problem posing. In I. Hirabayashi, N. Nohda, K. Shigematsu y F. L. Lin (Eds.), Proceedings of the Seventeenth International Conference for the Psychology of Mathematics Education (Vol. 3, pp. 41-47). Tsukuba (Japan): International Group for the Psychology in Mathematics Education.

Muñoz, I. (1994). El pensamiento creativo. Desarrollo del programa “Xenius". Barcelona: Octaedro.

Nadjafikhah, M., \& Yaftian, N. (2013). The frontage of Creativity and Mathematical Creativity. Procedia-Social and Behavioral Sciences, 90, 344-350.

National Advisory Committee on Creative and Cultural Education (NACCCE) (1999). All Our Futures: Creativity, Culture and Education. London: Department for Education and Employment. 
Nicolaou, A., \& Pilippou, G. (2007). Eficacy belief, problem posing, and mathematics achievement. En D. Pitta-Pantazi, y G. Philippou (Eds.), Proceedings of the V Congress of the European Society for Research in Mathematics Education (pp. 308-317). Larnaca (Chipre): Department of Education, University of Cyprus.

Noda, A. (2000). Aspectos epistemológicos y cognitivos de la resolución de problemas de matemáticas, bien y mal definidos. Un estudio con alumnos del primer ciclo de la ESO y maestros en formación (Tesis doctoral inédita). Universidad de la Laguna, España.

Pichel, J. (2001). Requeteoremas: reinventando teoremas en geometría con Cabri II. Suma, 36, 17-22.

Pintér, K. (2012). On Teaching Mathematical Problem-Solving and Problem Posing. (Tesis doctoral inédita). University of Szeged, Hungary.

Poincaré, H. (1908). Science et méthode. París: Flammarion.

Polya, G. (1965). Cómo plantear y resolver problemas. México DF: Trillas.

Puig, L. (1993). Elementos para la instrucción en resolución de problemas de matemáticas (Tesis doctoral inédita). Universitat de Valencia, España.

Ramos, M. (2006). Educadores creativos, alumnos creadores: Teoría y práctica de la creatividad. Caracas: San Pablo.

Sequera, E. C. (2007). Creatividad y desarrollo profesional docente en matemáticas para la educación primaria (Tesis doctoral inédita). Universidad de Barcelona, España.

Schroeder, T. L., \& Lester, F. K. (1989). Developing understanding in mathematics via problem solving. En P. R. Trafton y A. P. Shulte (Eds.), New directions for elementary school mathematics (pp. 31-42). Reston, VA: The Council.

Sheikhzade, M. (2008). Promoting skills of problem-posing and problemsolving in making a creative social studies classroom. Presentado en la 4th Global Conference, Oxford.

Shukkwan, S. L. (1993). Mathematical problem posing: The influence of task formats, mathematics knowledge, and creative thinking. In I. Hirabayashi, N. Nohda, K. Shigematsu y F. L. Lin (Eds.), Proceedings of the 17th International Conference for the Psychology of Mathematics 
Education (vol. 3, pp. 33-40). Tsukuba (Japan): International Group for the Psychology in Mathematics Education.

Silver, E. A. (1994). On mathematical problem posing. For the Learning of Mathematics, 14(1), 19-28.

Silver, E. A. (1997). Fostering creativity through instruction rich in mathematical problem solving and problem posing. ZDM, 29(3), 75-80.

Silver, E. A., \& Cai, J. (1996). An analysis of arithmetic problem posing by middle school students. Journal for Research in Mathematics Education, 27(5), 521-539.

Silver, E., Mamona-Downs, J., Leung, S., \& Kenney, P. (1996). Posing mathematical problem: an exploratory study. Journal for research in matehematics education, 27(3), 293-309.

Singer, F. M., Perczer, I., \& Voica, C. (febrero, 2011). Problem posing and modification as a criterion of mathematical creativity. Trabajo presentado a The Seventh Congress of the European Society for Research in Mathematics Education, Universidad de Rzeszów, Polonia. Trabajo recuperado de http://www.cerme7.univ.rzeszow.pl/WG/7/Singer_et-al_ CERME7_WG7.pdf.

Skinner, P. (1991). What's your problem? Posing and solving mathematical problems, $K-2$. Portsmouth, NH: Heinemann.

Song, S., Yim, J., Shin, E., \& Lee, H. (2007). Posing problems with use the 'what if not?' strategy in NIM game. En J. H. Woo, H. C. Lee, K. S. Park y D. Y. Seo (Eds.), Proceedings of the $31^{\text {st }}$ Conference of the International Group for the Psychology of Mathematics Education (Vol. 4, pp. 193-200). Seúl, Corea del Sur: PME.

Sorin, M. (1992). Creatividad. ¿Cómo, por qué, para qué? Barcelona: Labor.

Stein, M.I. (1956). A Transactional approach to creativity. En D. W. Taylor (Ed.), Research conference on the identification of creative talent. Salt Lake City: University of Utah Press.

Stoyanova, E. (1998). Problem posing in mathematics classrooms. En A. McIntosh y N. Ellerton (Eds.), Research in Mathematics Education: a contemporary perspective (pp. 164-185). Edit Cowan University: MASTEC. 
Stoyanova, E., \& Ellerton, N. F. (1996). A framework for research into students' problem posing in school mathematics. En P. C. Clarkson (Ed.), Technology in mathematics education (pp. 518-525). Melbourne, Victoria: Mathematics Education Research Group of Australasia.

Streefland, L. (1987). Free production of fraction monographs. En J. C. Bergeron, N. Herscovics y C. Kieran (Eds.), Proceedings of the Eleventh Annual Meeting of the International Group for the Psychology of Mathematics Education (Vol. 1, pp. 405-410. Montreal: Canada.

Van den Brink, J. F. (1987). Children as arithmetic book authors. The learning of mathematics, 7(2), 44-48.

Van Harpen, X. Y., \& Sriraman, B. (2013). Creativity and mathematical problem posing: an analysis o high school student's mathematical posing in China and the USA. Educational Studies Mathematics, 82(2), 201-221.

Whitin, D. J. (2006). Problem posing in the elementary classroom. Teaching Children Mathematics, 13(1), 14-18.

Vianney, J. P. y Navarro, A. (2011). Solución de problemas, creatividad e innovación. BuenasTareas.com. Recuperado de http://www. buenastareas.com/ensayos/Solucion-De-Problemas-Creatividad-eInnovacion/3096644.html. 